following manner. Readings as outlined above in 2, 3, and 4 were taken in a dark room in the direct paths of radiation from an electric heater, and of wind from a fan, and it was possible by means of these observations and previous calibrations, to predict successfully the readings corresponding to the cases when either the heater or the fan or both of them were removed.

The applications of this method are numerous. In particular it should be possible to allow completely for the influence of solar radiation and atmospheric turbulence upon the readings of various instruments which are at present considered unreliable under open air conditions. The comparative analysis of heating by radiation and heating by convection has important physical bearings and may be facilitated by measurements of this kind.

McGill University.

Geometrical Proof for the Wadsworth Constant Deviation System.

By R. C. Gibbs and J. R. Collins.

By an analytical method Wadsworth, ${ }^{1}$ determined the location of the axis of rotation of a constant-deviation prism-mirror system such that the emergent ray remains fixed in position as the system is rotated. This proof involves a series of long though simple transformations.

The location of this axis can be determined by a short geometrical proof similar to that used by E. Bloch, ${ }^{2}$ in finding the corresponding axis of rotation for a constant deviation prism of the Pellin-Broca type.

CORNell. University,

December, 192 I.

\title{
A Piezo-Electric Method for Generating Electric Oscillations of Constant Frequency.
}

By W. G. CaDy.

IN a former communication ${ }^{3}$ several methods were described whereby the frequency of a vacuum-tube oscillating circuit could be rendered very stable by the use of a piezo-electric resonator of proper dimensions. Further investigation has shown that it is possible, with the aid of amplifiers, to make the piezo-electric resonator serve as a mechanically tuned feedback, so that a highfrequency alternating current is generated, whose frequency is determined solely by the mechanical vibrations of the quartz plate. The plate is so mounted as to vibrate freely longitudinally between two pairs of close-fitting metallic "coatings." The first pair of coatings is connected between the output anode of a three-stage resistance amplifier and ground, while the second pair is connected between the input grid and ground.

1 Astronomy and Astro Physics, Vol. I3, p. 835, 1894.

${ }^{2}$ Journal de Physique, Vol. 7, p. I45, I9r7.

${ }^{3}$ Physical Review, I8, p. I42, I92r. A more complete account of these experiments, together with the theory of the piezo-electric resonator, will appear $n$ the Proceedings of the Institute of Radio Engineers. 